\title{
A Vernier Time-to-Digital Converter With Delay Latch Chain Architecture
}

\author{
Niklas Andersson and Mark Vesterbacka
}

\section{Linköping University Post Print}

\section{Tweet}

N.B.: When citing this work, cite the original article.

Niklas Andersson and Mark Vesterbacka, A Vernier Time-to-Digital Converter With Delay Latch Chain Architecture, 2014, IEEE Transactions on Circuits and Systems - II - Express Briefs, (61), 10, 773-777.

http://dx.doi.org/10.1109/TCSII.2014.2345289

C2014 IEEE. Personal use of this material is permitted. However, permission to reprint/republish this material for advertising or promotional purposes or for creating new collective works for resale or redistribution to servers or lists, or to reuse any copyrighted component of this work in other works must be obtained from the IEEE.

\section{http://ieeexplore.ieee.org/}




\title{
A Vernier Time-to-Digital Converter with Delay Latch Chain Architecture
}

\author{
Niklas U. Andersson, Student Member, IEEE, and Mark Vesterbacka, Senior Member, IEEE
}

\begin{abstract}
A new Vernier time-to-digital converter architecture using a delay line and a chain of delay latches is proposed. The delay latches replace the functionality of one delay chain and the sample register commonly found in Vernier converters, hereby enabling power and hardware efficiency improvements. The delay latches can be implemented using either standard or full custom cells allowing the architecture to be implemented in FPGAs, digital synthesized ASICs, or in full custom design flows. To demonstrate the proposed concept, a 7-bit Vernier time-todigital converter has been implemented in a standard $65 \mathrm{~nm}$ CMOS process with an active core size of $33 \mu \mathrm{m} \times 120 \mu \mathrm{m}$. The time resolution is $5.7 \mathrm{ps}$ with a power consumption of $1.75 \mathrm{~mW}$ measured at a conversion rate of $100 \mathrm{MS} / \mathrm{s}$.
\end{abstract}

Index Terms-Time-to-digital converter, TDC, delay latch, Vernier, CMOS

\section{INTRODUCTION}

$\mathbf{I}$ $\mathrm{N}$ recent years, time domain signal processing has become a promising alternative to signal processing implemented in the voltage or current domains. The reason is that the intrinsic gain of the CMOS transistors, that is transconductance over channel conductance $g_{m} / g_{d s}$, is reduced for each new CMOS process node [1]. At the same time the cut-off frequency, $f_{t}$, for the same CMOS transistors increase. As a result of this, the resolution in the voltage domain decreases while the resolution in the time domain increases.

Time-to-digital converters (TDCs) are for example used in analog-to-digital converters [2], [3] and in digital phase-locked loops (PLLs) as a replacement for the phase comparator [4]. By replacing the phase comparator with a TDC, the charge pump and the analog loop filters can be replaced with digital filters and a digital control loop. In the PLL case, the inputs to the TDC are two clock signals which is the notation we will use in this work.

In this work we propose a new Vernier TDC architecture enabling both power and area improvements. These savings are made possible by replacing the second delay chain and the sampling register commonly found in Vernier converters with a chain of delay latches.

The proposed architecture can be implemented using either digital standard cells or full custom cells. Even though the proposed architecture can be implemented as a single delayline TDC we will use the delay lines in a Vernier configuration in this work. The Vernier configuration is used since a single delay line TDC is limited by the gate delay while the Vernier TDC can achieve sub-gate delay resolution [5], [6]. All transistors in the design are used as digital switches, hence the proposed architecture suits for implementation in CMOS processes with reduced feature size.

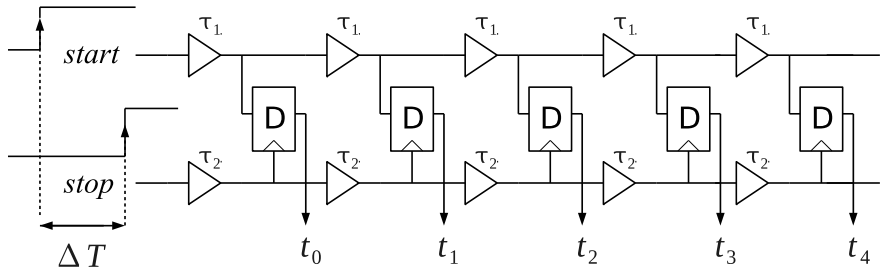

Fig. 1. Illustration of a common delay line Vernier TDC.

A hardware-efficient reset and edge detect circuit is proposed. The circuit generates a reset before each conversion cycle and also filters out the correct edges of the input signals. The circuit can also handle input signals with different signal frequencies.

The paper is organized as follows. Section II describes the delay latch chain TDC architecture and the reset and edge detect circuit. Section III presents measurement results and comparisons with recently published TDCs. The paper is concluded in Section IV.

\section{Proposed TDC ARChitecture}

A TDC converts the time difference between two input signals to a digital output word. In a single delay line TDC the time resolution is limited by the gate delay in the delay chain whereas a Vernier TDC can achieve sub gate-delay resolution [5], [6]. A Vernier TDC compares the delays of two delay lines by sampling the state of one delay line with a signal that has propagated through a second delay line with a shorter unit delay, as illustrated in Fig. 1. Assuming that the unit delays of the start and stop delay lines are $\tau_{1}$ and $\tau_{2}$ respectively, the resolution of a Vernier TDC is given by the delay difference $\tau_{\mathrm{LSB}}=\tau_{1}-\tau_{2}$.

The proposed Vernier architecture is described in Sec. II-A and the reset and edge filtering circuit is described in Sec. II-B.

\section{A. Delay latch chain}

The proposed TDC architecture consisting of a chain of delay latches with unit delays $\tau_{1}$ and a delay line with unit delays $\tau_{2}$ is illustrated in Fig. 2. The delay latches are transparent if the control input is low and they hold their output values if the control input is high. The delay latches are modeled using buffers and multiplexers with zero delay connected in feedback. A complete conversion cycle for the proposed architecture in Fig. 2 consists of the following steps where it is assumed that $\tau_{1}>\tau_{2}$.

1) The TDC is prepared for conversion in the reset phase where the start and stop inputs are low. All delay latches are now transparent. 


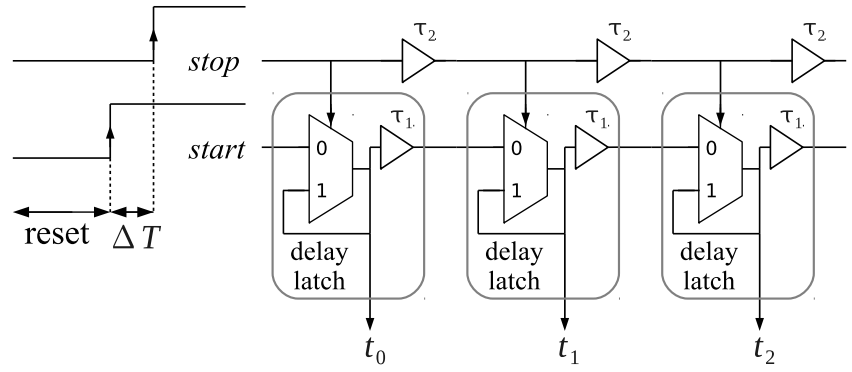

Fig. 2. Illustration of the proposed delay latch chain Vernier TDC.

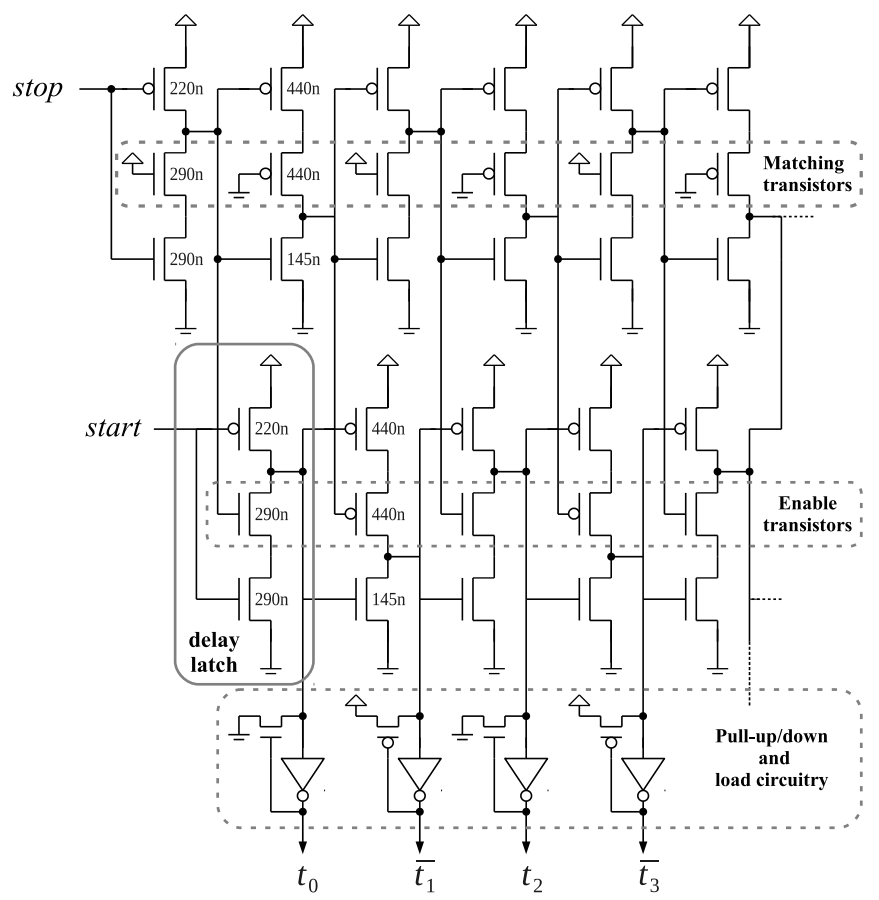

Fig. 3. Detailed implementation of the proposed TDC.

2) At the next rising edge of the start input, a pulse propagates through the delay latch chain gradually increasing the thermometer code at the $t_{x}$ outputs.

3) At the next rising edge of the stop input, a second pulse propagates through the delay line continuously setting the delay latches in hold state.

4) When the stop pulse catches up with the start pulse, the $N$ th delay latch is non-transparent hereby stopping the propagation of the start pulse.

5) The thermometer code, $t_{x}$, at the output of the delay latches is now linearly dependent on the time difference, $\Delta T$, between the two inputs.

The delay latches in the proposed architecture can be implemented in a variety of ways using either standard cells or a full custom solution. A hardware-efficient circuit is illustrated in Fig. 3 where the delay latch chain is implemented using dynamic inverters with alternating NMOS and PMOS enable transistors and works as follows.

When the gate voltage is set high on an NMOS enable transistor the delay latch works as an inverting delay element and when the gate voltage is low the output of the delay latch

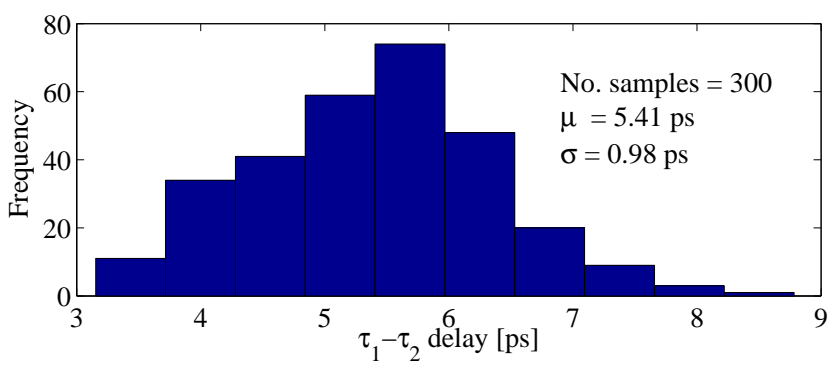

Fig. 4. Histogram showing the simulated unit delay difference between the delay latch chain and the delay line, $\tau_{\mathrm{LSB}}=\tau_{1}-\tau_{2}$.

becomes a floating node hence holding the current voltage value. The PMOS enable transistors works in the same way as the NMOS transistors but with complementary gate voltages.

Since the delay latch outputs, $t_{x}$, are floating when the enable transistors are turned off, that is, no path exists to supply nor ground, pull-up/down circuitry are added as illustrated in Fig. 3. The pull-up/down circuitry have two additional purposes, that is acting as an extra load to ensure that $\tau_{1}>\tau_{2}$, and also act as buffers driving the inputs of the thermometerto-binary encoder.

Matching transistors are added to the delay line inverters to match PVT variations. The matching transistors are always enabled by connecting the NMOS and PMOS transistor gates to supply and ground potentials, respectively. Note that all delay latches and delay elements are inverting in the detailed implementation, hence every second thermometer code bit is also inverted. This can however easily be corrected for in the succeeding thermometer-to-binary encoder.

Each delay stage requires nine transistors including the pull-up/down circuitry. This can be compared to the standard Vernier TDC architecture in Fig. 1 that requires 28 transistors per delay stage in an implementation assuming that one D flipflop uses 24 transistors. Hence the proposed solution reduces the transistor count by $68 \%$.

Monte Carlo simulations have been performed on an extracted layout made in a $65 \mathrm{~nm}$ CMOS process to predict how the delay difference $\tau_{1}-\tau_{2}$ is affected by the process variations. The supply voltage is $1.2 \mathrm{~V}$ and the temperature $70^{\circ} \mathrm{C}$. From the histogram in Fig. 4 it can be concluded that the TDC has an expected time resolution of 5.4 ps with a variance of $1.0 \mathrm{ps}$ due to process variations and transistor mismatch. If a smaller variance is required, calibration can be applied as for example suggested in [7].

Figures 5 (a)-(c) show simulated integrated non-linearity (INL) results for three process corners, that is the typical, the fast NMOS/slow PMOS, and the slow NMOS/fast PMOS corners. The differential non-linearity (DNL) for the same corners are shown in Figs. 6 (a)-(c). The simulations show that the linearity of the TDC is stable over process corners but there is a spread in time resolution as was also seen in Fig. 4. In Figs. 5 (a)-(c), we find a large drop in INL for lower-end codes. This is caused by an insufficiently sized inverter (INV1 in Fig. 7 (b)) as is further discussed in Sec. III-A. A simulation with a correctly sized inverter is shown in Fig. 5 (d). 


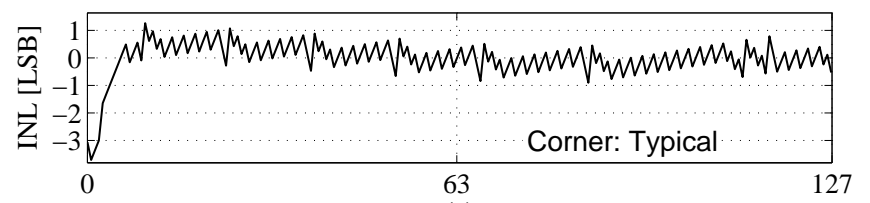

(a)

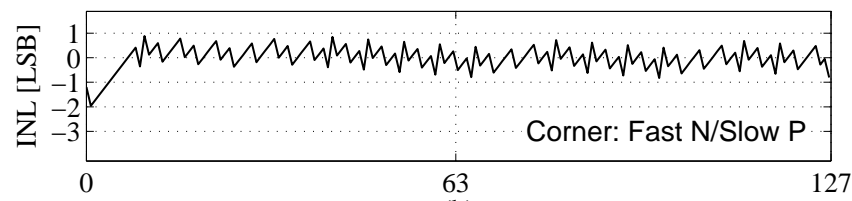

(b)

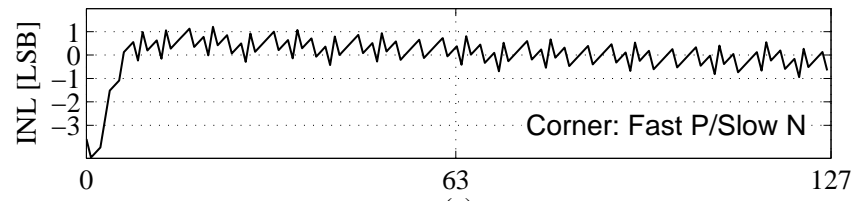

(c)

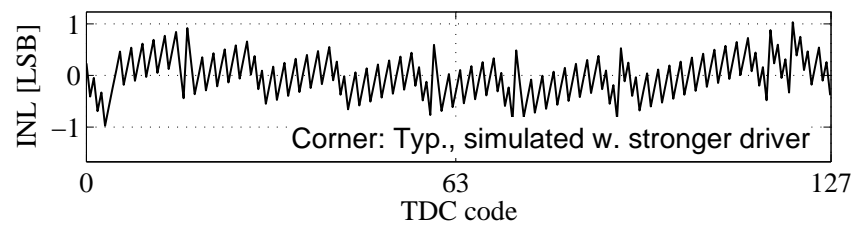

(d)

Fig. 5. INL simulations for (a) typical process corner, (b) fast N/slow P, (c) slow N/fast P, and (d) typical corner with a correctly sized inverter. Resolutions are $5.5,4.7,4.5$ and $5.5 \mathrm{ps} / \mathrm{LSB}$ respectively.

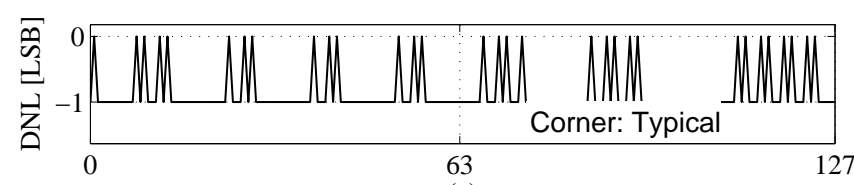

(a)

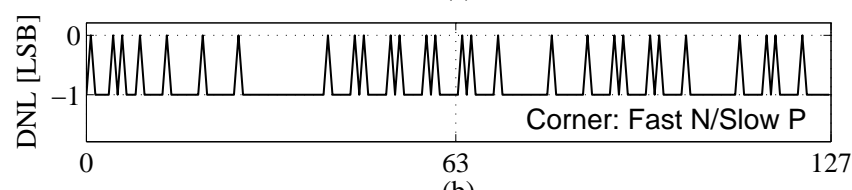

(b)

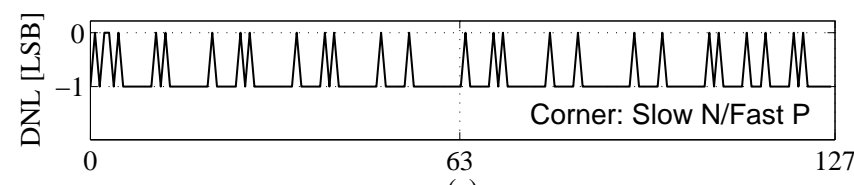

(c)

Fig. 6. DNL simulations for (a) typical, (b) fast N/slow $\mathrm{P}$, and (c) slow N/fast $\mathrm{P}$ process corners. Resolutions are 5.5, 4.7, and $4.5 \mathrm{ps} / \mathrm{LSB}$ respectively.

\section{B. Reset and edge detection circuit}

The TDC requires a reset before each conversion cycle and should also measure the time difference between the rising edge of $c l k A$ and the next rising edge of $c l k B$ as shown in the timing diagram in Fig. 7 (c).

A high level description of a circuit generating a reset before each conversion and also performing the edge detection is illustrated in Fig. 7 (a) where the $c l k A$ and $c l k B$ are the inputs to the circuit and the start and stop signals are inputs to the succeeding Vernier TDC.

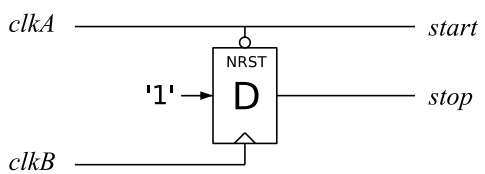

(a)

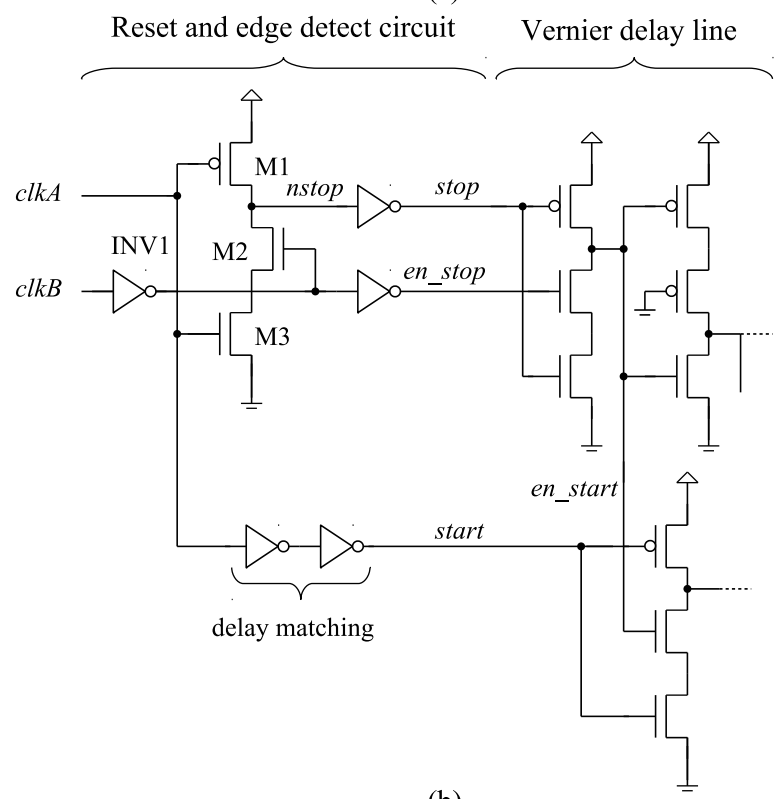

(b)

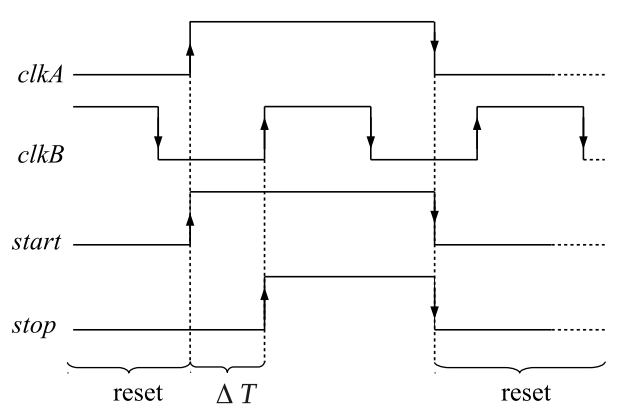

(c)

Fig. 7. Illustrations of (a) a high level description, (b) circuit implementation, and (c) timing diagram of the reset and edge detection circuit.

An efficient implementation of the circuit in Fig. 7 (a) is shown in Fig. 7 (b). The circuit uses less hardware than the D flip-flop implementation in Fig. 7 (a), which makes it easier to maintain a constant delay between the $c l k A$ and $c l k B$ inputs.

The circuit in Fig. 7 (b) works as follows. When clkA is low, both delay lines are reset by discharging the start and stop nodes. The en_start is now charged allowing a pulse to ripple through the delay latch chain at the next rising edge of the start node. At the rising edge of clkA the start node is charged high and the delay latch chain starts to ripple. At the same time, the nstop node is discharged through transistors M2 and M3 thus charging the stop input. However, since the en_stop node is still low, the stop delay line will not start to ripple until the next rising edge of $c l k B$.

\section{Thermometer-to-binary encoder}

The thermometer-to-binary encoder is a crucial building block since it accounts for approximately $60 \%$ of the total 


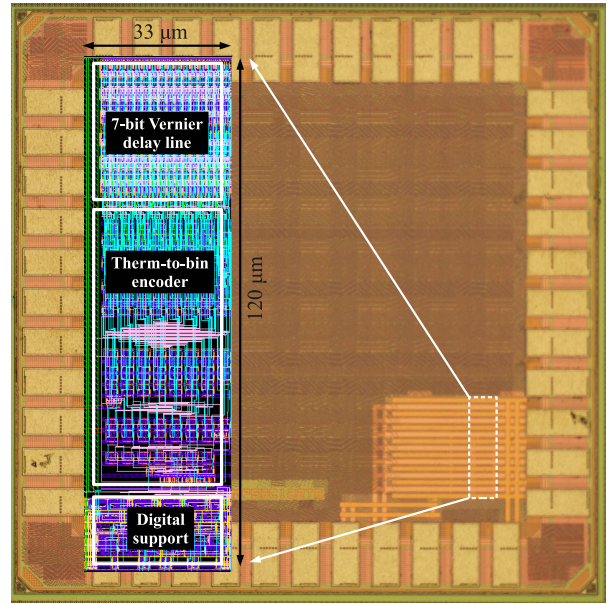

Fig. 8. Chip photo of the 7-bit TDC.

dynamic power consumption of this power efficient TDC. A power split for the implemented TDC is given in Sec. III-B. To minimize the power consumption an encoder based on multiplexers was chosen. Previous investigations show that such an encoder [8], [9] requires less hardware, has a shorter critical path and also lower power consumption as compared to commonly used one's-counter solutions.

\section{Measurements}

The TDC was implemented in a standard $65 \mathrm{~nm}$ CMOS process with a core size of $33 \mu \mathrm{m} \times 120 \mu \mathrm{m}$. A chip photo is shown in Fig. 8 and the measurement results from four chip samples are presented below.

\section{A. Time resolution and single shot precision}

The time resolution was measured using a Rohde Schwarz SMBV100A vector signal generator where the I and Q outputs from the RF baseband generator were used as inputs to the TDC. The time difference between the input signals was swept in $1 \mathrm{ps}$ steps and $10 \mathrm{kSamples}$ were collected for each of the settings. The average of these samples was derived and the resulting differential and integrated non-linearity, INL and DNL, curves are shown in Fig. 9. The DNL and INL curves are normalized to the average time resolution of the TDC which was measured to $5.7 \mathrm{ps}$.

From the DNL curve in Fig. 9 (a) it is seen that the TDC is monotonic. Monotonicity is important if the converter is used in closed loop applications such as for example digital phase-locked loops [5].

In Fig. 9 (b) and (c) the INL was derived using the bestfit line and the end-to-endpoint definitions respectively. In Fig. 9 (b) we find the INL to be -5 LSBs for lower-end codes. This comparatively high non-linearity is caused by an insufficiently sized inverter, INV1 in Fig. 7 (b). The relatively long rise time of the inverter unfortunately sets the latch (i.e., the path through transistors M1, M2 and M3) in a metastable state for low input codes, which is when the rising edges of clkA and $c l k B$ are close in time. The metastability increases the delay through the latch resulting in the non-linear INL

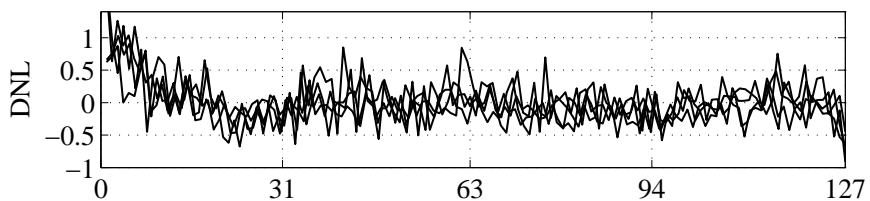

(a)

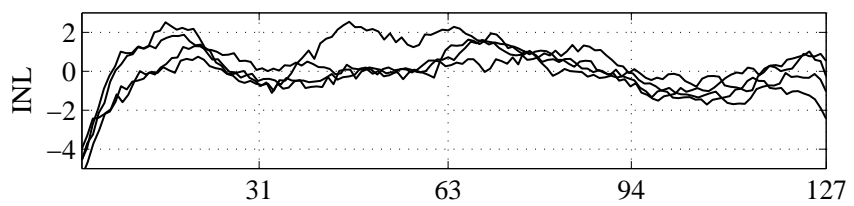

(b)

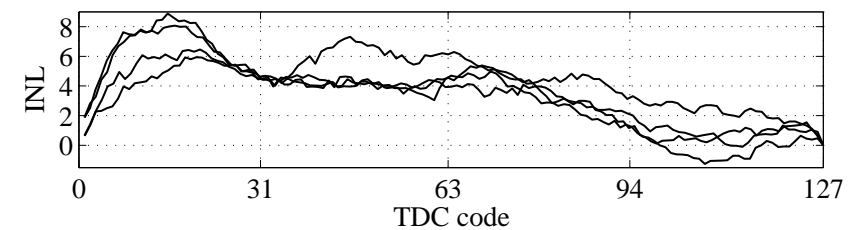

(c)

Fig. 9. Measurement results (normalized to $5.7 \mathrm{ps)} \mathrm{from} \mathrm{four} \mathrm{chip} \mathrm{samples}$ of (a) DNL, (b) INL derived using the best-fit line definition, and (c) INL derived using the end-to-endpoint definition.

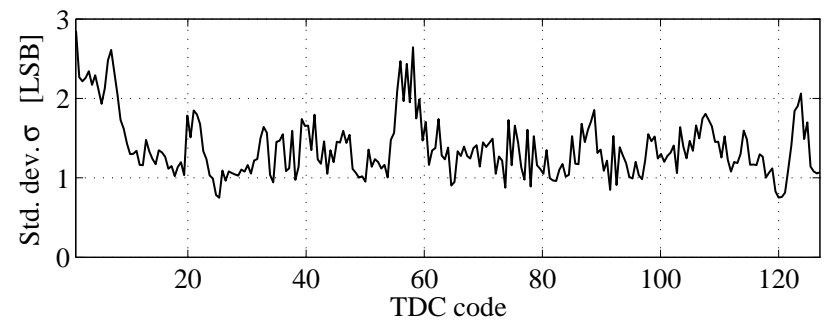

Fig. 10. Measured single shot standard deviation as a function of TDC code.

curve. Careful simulations verify this hypothesis and an INL simulation with a correctly sized inverter is shown in Fig. 5 (d). For codes higher than 6 , INL is within \pm 2.5 LSBs. If the endto-endpoint INL definition is used the worst case INL increases to 9 LSBs as seen in Fig. 9 (c).

The single shot precision measures the output of the converter for a constant input signal. This measurement catches noise and other non-ideal behavior from on-chip as well as off-chip sources. The time difference of the input signals was swept in 1 ps steps and $10 \mathrm{kSamples}$ were sampled for each setting. The standard deviation $\sigma$ was derived for each input code and is plotted in Fig. 10. The variation in standard deviation probably originates from a non-uniform layout of the TDC. Histograms for three selected input codes are shown in Fig. 11.

\section{B. Power consumption and conversion rate}

The maximal conversion rate was measured to $100 \mathrm{MS} / \mathrm{s}$. The total power consumption at this conversion rate was $1.75 \mathrm{~mW}$ of which $20 \%$ are consumed in the Vernier chain, $60 \%$ in the thermometer-to-binary encoder, $10 \%$ in the digital support block and $10 \%$ in the output buffers driving the digital I/Os. The power consumption as a function of the conversion rate is plotted in Fig. 12. 

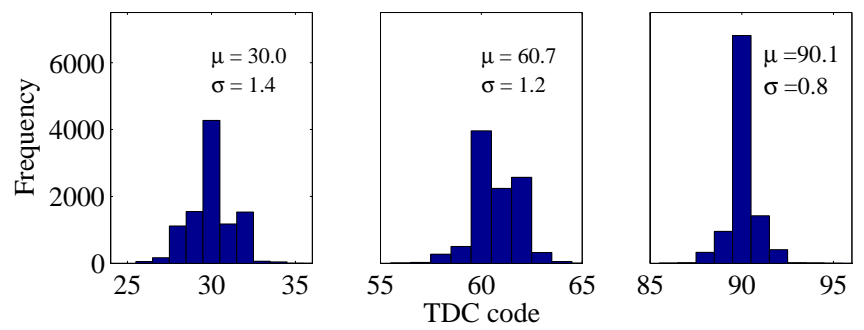

Fig. 11. Histograms from single shot precision measurements for three different input codes.

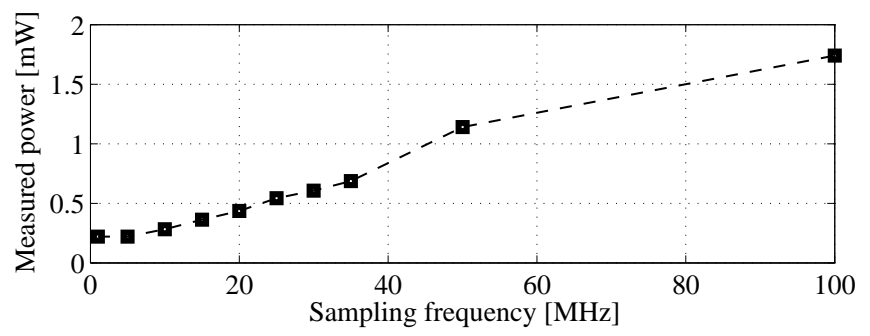

Fig. 12. Measured power consumption versus conversion rate.

\section{Comparison with recently published TDCs}

In Table I the implemented TDC is compared with recently published TDCs with a resolution in the range 4-6 ps. The TDCs in Table I are selected with respect to small area and low power consumption. Note that there are converters with sub ps resolution [10], [11]. The finer time resolution does however come with a significantly larger chip area and power consumption.

From Table I it can be concluded that the proposed TDC offers competitive performance in terms of area and power consumption. The delay line TDC has shorter conversion range than a looped architecture [12]. Intended application areas for the proposed TDC are counter-assisted digital PLLs [5] and all-digital ADCs [2], [13].

The limited measured non-linearity will be addressed in future designs by mainly resizing the inverter in the edge detect circuit as described in Sec. III-A. Note that the prototype chip still shows a high potential of the proposed architecture.

\section{CONCLusions}

A new Vernier TDC architecture using a delay line and a chain of delay latches has been presented. It has been demonstrated that a full custom implementation of the proposed architecture reduces the transistor count with $68 \%$ as compared to a conventional solution leading to both power and area savings. A 7-bit Vernier TDC has been implemented in a standard $65 \mathrm{~nm}$ CMOS process with an active core size of $33 \mu \mathrm{m} \times 120 \mu \mathrm{m}$. The time resolution was measured to $5.7 \mathrm{ps}$ with a power consumption of $1.75 \mathrm{~mW}$ at a conversion rate of $100 \mathrm{MS} / \mathrm{s}$.

\section{REFERENCES}

[1] F. Ellinger, M. Claus, M. Schroter, and C. Carta, "Review of advanced and beyond CMOS FET technologies for radio frequency circuit design," in IEEE MTT-S International Microwave Optoelectronics Conference (IMOC), 2011, pp. 347-351.
[2] J. Daniels, W. Dehaene, M. Steyaert, and A. Wiesbauer, "A/D conversion using asynchronous Delta-Sigma modulation and time-to-digital conversion," IEEE Trans. Circuits Syst. I, vol. 57, no. 9, pp. 2404-2412, 2010.

[3] M. M. Elsayed, V. Dhanasekaran, M. Gambhir, J. Silva-Martinez, and E. Sanchez-Sinencio, "A 0.8 ps DNL time-to-digital converter with $250 \mathrm{MHz}$ event rate in $65 \mathrm{~nm}$ CMOS for time-mode-based modulator," IEEE J. Solid-State Circuits, vol. 46, no. 9, pp. 2084-2098, 2011.

[4] T. Rahkonen and J. Kostamovaara, "Low-power time-to-digital and digital-to-time converters for novel implementations of telecommunication building blocks," in Proc. IEEE Int. Symp. Circuits Syst., vol. 3, 1994, pp. 141-144.

[5] S. Henzler, Time-to-Digital Converters, ser. Springer Series in Advanced Microelectronics. Springer, 2010.

[6] T. E. Rahkonen and J. T. Kostamovaara, "The use of stabilized CMOS delay lines for the digitization of short time intervals," IEEE J. SolidState Circuits, vol. 28, no. 8, pp. 887-894, 1993.

[7] P. Das and B. Amrutur, "An accurate fractional period delay generation system," IEEE Trans. Instrum. Meas., vol. 61, no. 7, pp. 1924-1932, 2012.

[8] E. Säll, "Implementation of flash analog-to-digital converters in siliconon-insulator CMOS technology," Ph.D. dissertation, 2007.

[9] P. Capofreddi, "Method and apparatus for low power thermometer to binary coder," U.S. Patent 6,542,104 B1, Apr. 1, 2003.

[10] M. Lee and A. A. Abidi, "A 9 b, 1.25 ps resolution coarse-fine time-todigital converter in $90 \mathrm{~nm}$ CMOS that amplifies a time residue," IEEE J. Solid-State Circuits, vol. 43, no. 4, pp. 769-777, 2008.

[11] Y.-H. Seo, J.-S. Kim, H.-J. Park, and J.-Y. Sim, "A 0.63ps resolution, $11 \mathrm{~b}$ pipeline TDC in $0.13 \mu \mathrm{m}$ CMOS," in IEEE Symposium on VLSI Circuits (VLSIC), 2011, pp. 152-153.

[12] M. Z. Straayer and M. H. Perrott, "A multi-path gated ring oscillator TDC with first-order noise shaping," IEEE J. Solid-State Circuits, vol. 44, no. 4, pp. 1089-1098, 2009.

[13] Q. Chen, Z. H. Shen, N. Yan, X. Tan, and H. Min, "Monolithic digitally controlled buck converter with TDC-based ADC sharing delay cells with DPWM," Electronics Letters, vol. 48, no. 20, pp. 1303-1304, 2012.

[14] S. Henzler, S. Koeppe, W. Kamp, H. Mulatz, and D. Schmitt-Landsiedel, "90nm 4.7ps-resolution 0.7-LSB single-shot precision and 19pJ-pershot local passive interpolation time-to-digital converter with on-chip characterization," in Proc. IEEE Int. Solid-State Circuit Conf., 2008, pp. 548-635.

[15] Y. Park and D. D. Wentzloff, "A cyclic Vernier TDC for ADPLLs synthesized from a standard cell library," IEEE Trans. Circuits Syst. I, vol. 58, no. 7, pp. 1511-1517, 2011.

[16] L. Vercesi, A. Liscidini, and R. Castello, "Two-dimensions Vernier timeto-digital converter," IEEE J. Solid-State Circuits, vol. 45, no. 8, pp. 1504-1512, 2010.

[17] P. Lu, A. Liscidini, and P. Andreani, "A $3.6 \mathrm{~mW}$, $90 \mathrm{~nm}$ CMOS gatedVernier time-to-digital converter with an equivalent resolution of $3.2 \mathrm{ps,"}$ IEEE J. Solid-State Circuits, vol. 47, no. 7, pp. 1626-1635, 2012.

TABLE I

RECENTLY PUBLISHED TIME-TO-DIGITAL CONVERTERS.

\begin{tabular}{lccccc}
\hline Type & $\begin{array}{c}{[14]} \\
\text { Passive } \\
\text { interp. }\end{array}$ & $\begin{array}{c}\text { Cyclic } \\
\text { Vernier }\end{array}$ & $\begin{array}{c}\text { [16] } \\
\text { 2-D } \\
\text { delay line }\end{array}$ & $\begin{array}{c}\text { Vernier } \\
\text { + GRO }\end{array}$ & $\begin{array}{c}\text { This } \\
\text { Work }\end{array}$ \\
\hline Samp. rate [MS/s] & 180 & 10 & 50 & $25 / 100$ & 100 \\
Resolution [ps] & & 5.5 & 4.8 & 5.8 & 5.7 \\
Oversampling ratio & 4 & & & 16 & \\
Res. w. interp. [ps] & 4.7 & & & 3.2 & \\
Power supply [V] & 1.2 & 1.0 & 1.2 & 1.2 & 1.2 \\
Power [mW] & 3.6 & 2.0 & $1.7^{a}$ & $3.6^{b}$ & $1.14 / 1.75^{c}$ \\
Range [ns] & 0.6 & 100 & 0.6 & 40 & 0.73 \\
Number of bits & 7 & 15 & 7 & & 7 \\
Area [mm ${ }^{2}$ ] & 0.02 & 0.006 & 0.02 & 0.027 & 0.004 \\
Technology [nm] & 90 & & 65 & 90 & 65 \\
Year & 2008 & 2011 & 2010 & 2012 & 2013 \\
\hline
\end{tabular}

${ }^{a}$ measured at $50 \mathrm{MS} / \mathrm{s}$

${ }^{b}$ measured at $25 \mathrm{MS} / \mathrm{s}$

${ }^{c}$ measured at 50/100 MS/s 